\title{
A Hybrid Event Trigger Mechanism and Time-Delay Partitioning Are Applied to Event-Driven Control Systems
}

\author{
WanRu Wang $(\mathbb{D})^{1,2}$ LianKun Sun, ${ }^{1,2}$ and BinBin Shen ${ }^{1}$ \\ ${ }^{1}$ School of Computer Science and Technology Engineering, Tiangong University, Tianjin 300387, China \\ ${ }^{2}$ Tianjin Key Laboratory of Autonomous Intelligence Technology and Systems, Tianjin 300387, China
}

Correspondence should be addressed to WanRu Wang; tjpuwwr@126.com

Received 8 April 2020; Accepted 22 June 2020; Published 1 August 2020

Guest Editor: Sahar Yazdani

Copyright (C) 2020 WanRu Wang et al. This is an open access article distributed under the Creative Commons Attribution License, which permits unrestricted use, distribution, and reproduction in any medium, provided the original work is properly cited.

This paper proposes an idea of using time-delay partitioning to construct a Lyapunov-Krasovskii functional (LKF) to analyse event-driven network control systems (NCSs) with the Ho performance. Firstly, select a mixed event-driven mechanism, in which an adjustable absolute trigger mechanism is added to the trigger condition. Trigger term can be indicated to use a delay model. Secondly, a suitable LKF is created, which makes use of time-delay partitioning. Based on Wirtinger inequality and linear matrix inequalities (LMI), the close system with $H \infty$ performance index level is global uniform ultimate bounded. Finally, a numerical simulation example proves the effectiveness of the proposed method.

\section{Introduction}

With the rapid development of computer and networked technologies, data transmission via communication networks has received considerable research attentions [1-10]. Due to the advantages of the NCSs such as data sharing, low cost, and easy maintenance, it has been widely used in the field of process control, electricity system, aircraft control systems, automobile controlling systems, etc. The traditional control system is based on time-driven, but for the NCSs with limited resources, if the control tasks are executed periodically based on fixed time points, this not only wastes limited computing and bandwidth resources but also may cause network-induced phenomena such as network congestion, data transmission delay, and data loss. As a nonuniform scheduling mechanism, event-driven can effectively solve the abovementioned problems, so it has become a hot research topic at present.

In event-driven control, only when the system reaches the trigger threshold set in advance can the control instruction be executed or the information be transmitted. Therefore, event-driven control can achieve similar or better control performance and reduce data transmission rate, thus saving the limited network bandwidth, computation, energy, and other resources in the system. As such, fruitful results based on different event-driven schemes are made in [11-20]. The triggering threshold has a great effect on the implementation of control task [11, 12]; however, because the threshold of the static triggering mechanism is fixed, it is difficult to adjust for external interference and environmental changes. In order to overcome the above drawbacks, a dynamic event-triggered scheme has been addressed in [13-15]. Besides that, a Ho controller is designed in [21], in which Markov jump systems are studied based on eventtriggered considering finite time. Rahnama et al. [22] consider the effects of network-induced time delays, signal quantization, and data loss and show L-2-stability and robustness for the control design. Gu et al. [23] consider an adaptive $H \infty$ filter, which is based on event triggered to solve the problem of decentralize in NCSs. Furthermore, in [24], observer-based fuzzy controller is proposed to stabilize the NCSs under event-triggered mechanism in [25]; the stabilization problem for nonlinear NCSs with a two-terminal event-triggered mechanism is concerned. It is worth noting that the LKF method is mainly used in the above research, which will bring certain conservativeness to the conclusion due to the different treatment methods for cross items. 
In order to reduce the conservativeness of the conclusion, the investigators bring forward two different research directions. One way is to choose a suitable LKF, and the other is to select a better scaling method in the process of dealing with the integral terms. Rich investigative achievements have been achieved by LKF picking [26-30]. In the early years, a simple LKF for fixed-delay systems was adopted. In order to make the LKF contain more system information, fixed time delay and variable delay are added to the system status in LKF [26]. Come up with the opinion of using time-delay partitioning to establish LKF [27]. More explore consequences have been generated in dealing with integral items. Use Jensen inequality to process integral items in event-driven NCSs [31]. Seuret and Gouaisbaut [32] put forward cinequality to deal with the integral terms in the study of NCSs stability, which can introduce additional system integral terms to bring more system information. This method not only includes the information in the Jensen inequality but also adds some integral terms of the state to the system.

Motivated by the above discussions, in this paper, we focus on the Ho performance analysis of network control system based on event trigger mechanism. The main contributions of this paper are summarized as follows. (i) Using mixed trigger mechanism to reduce network burden, that is, an adjustable parameter is added on the basis of the dynamic trigger mechanism, so as to avoid Zeno phenomenon and long-term nonsampling phenomenon. (ii) The LKF is constructed with the idea of time-delay partitioning, and a new method is used to remove the coupling between the input matrix and the output matrix. On this basis, Wirtinger inequality is used to scale down and reduce the conservativeness of the conclusion.

1.1. Notation. In this paper, $Y>0(Y<0)$ denotes that the symmetric matrix $Y$ is positive (or negative). $\mathbb{R}^{n}$ is defined as $n$-dimensional Euclidean space. $\mathbb{R}^{n \times m}$ is defined as a set of $n \times m$ real matrix, $X^{T}$ refers to the transpose of $X, *$ denotes a symmetric term of a symmetric matrix, and $\|\cdot\|$ refers to the Euclidean norm. Followed by $M+M^{T}$, which is defined as $\mathrm{He}(M) . I_{n}$ refers to the $n$-dimensional unit matrix; $0_{n \times m}$ refers to the $n \times m$ dimension block matrix. The rest of the paper is adapted to the needs of the text of the adaptive dimension matrix.

\section{Problem Formulation}

Consider the following kind of linear time invariant system as follows:

$$
\left\{\begin{array}{l}
\dot{x}(t)=A x(t)+B_{1} u(t)+C_{1} \omega(t) \\
z(t)=D_{1} x(t)+B_{2} u(t)+C_{2} \omega(t) \\
y(t)=D_{2} x(t)
\end{array}\right.
$$

where $x(t) \in \mathbb{R}^{m}$ is the state vector, $u(t) \in \mathbb{R}^{n}$ is the control input, $y(t) \in \mathbb{R}^{p}$ is the control output, $z(t) \in \mathbb{R}^{q}$ is the control regulated output, $\omega(t) \in L_{2}[0, \infty]$ is the square integrable disturbance input, $A, B_{1}, B_{2}, C_{1}, C_{2}, D_{1}$, and $D_{2}$ are any matrices with adaptive dimensions. The eventtriggered communication mechanism can be described by

$$
\begin{gathered}
t_{k+1} h=t_{k} h+\min _{l}\left\{l h \mid\left\{e^{T}\left(i_{k} h\right) \Phi e\left(i_{k} h\right)\right.\right. \\
\left.\left.\geq \delta y^{T}\left(t_{k} h\right) \Phi y\left(t_{k} h\right)+\vartheta(t)\right\}\right\},
\end{gathered}
$$

where $\vartheta(t)=\beta \varepsilon^{-\alpha t}, e\left(i_{k} h\right)=y\left(i_{k} h\right)-y\left(t_{k} h\right)$ is the error between the output at the current sampling time $i_{k} h=t_{k} h+$ $\ln (l \in \mathbb{N})$ and the output at the latest triggered time $t_{k} h ; \Phi$ is a symmetric positive matrix; $h$ is the time sampling period; and $\delta \in[0,0.5]$ and $\beta \in[0,0.5]$ are given scalar. If $\delta=0$, the system trigger threshold is the absolute trigger mechanism. If $\beta=0$, the system trigger threshold is the relative trigger mechanism. If $\delta>0$ and $\beta>0$, the system trigger threshold is the mixed trigger mechanism.

Divide $\Omega$ into subsets $\Omega_{1}=\left[i_{k} h+d_{i k}, i_{k} h+h+d_{i k+1}\right)$, i.e., $\Omega=\cup \Omega_{1}$, where $i_{k} h=t_{k} h+l h, l=0, \ldots, t_{k+1}-t_{k}-1$; $i_{k} h$ and $t_{k} h$ represent the current sampling time and triggering time, respectively. For $l=t_{k+1}-t_{k}-1$, then $d_{i_{k+1}}=d_{t_{k+1}}$; otherwise, $d_{i_{k}}=d_{t_{k}}$. Define $\mathrm{d}(t) \triangleq t-i_{k} h$. Note that $h_{1} \leq \mathrm{d}(t) \leq h_{2}, h_{1}=d_{m}, h_{2}=d_{M}$, and $h_{m}=\left(h_{1}+h_{2}\right) / 2$. $h_{1}$ and $h_{2}$ represent the lower and upper bounds of the time delay $\left(h_{1}<h_{m}<h_{2}\right)$ :

$$
\left\{\begin{array}{l}
\dot{x}(t)=A x(t)+B_{1} K D_{2} x(t-\mathrm{d}(t))-B_{1} K e\left(i_{k} h\right)+C_{1} \omega(t), \\
z(t)=D_{1} x(t)+B_{2} K D_{2} x(t-\mathrm{d}(t))-B_{2} K e\left(i_{k} h\right)+C_{2} \omega(t), \\
u(t)=K x\left(t_{k} h\right), \quad t \in \Omega
\end{array}\right.
$$

where $u(t)$ is the controller law and control gain $K$.

Lemma 1 (see [12]). Given matrices $D, E(t)$, and $F$ of appropriate dimensions with $E(t)$ satisfying $E^{T}(t) E(t) \leq I$, for any $\varepsilon>0$, the following inequality holds:

$$
D E(t) F+F^{T} E^{T}(t) D^{T} \leq \varepsilon D D^{T}+\varepsilon^{-1} F^{T} F .
$$

Lemma 2 (see [12]). The following two inequalities are equivalent:

(a) There exists a symmetric and positive-definite matrix $P$ satisfying

$$
\left[\begin{array}{cc}
-P & A^{T} \\
A & -P^{-1}
\end{array}\right]<0 .
$$

(b) There exists a symmetric and positive-definite matrix $P$ and matrix $Y$ satisfying

$$
\left[\begin{array}{cc}
-P & (\mathrm{YA})^{T} \\
\mathrm{YA} & \mathrm{He}(-Y)+P
\end{array}\right]<0 .
$$

Lemma 3 (see [12]). For a given matrix $R>0$, the following inequality holds for all continuously differentiable function $\omega$ in $[a, b] \longrightarrow \mathbb{R}^{p}$ : 


$$
\begin{aligned}
\int_{a}^{b} \omega^{T}(u) R \omega(u) \mathrm{d} u \geq & \frac{1}{b-a}\left((\omega(b)-\omega(a))^{T} R(\omega(b)-\omega(a))\right) \\
& +\frac{3}{b-a} \mathfrak{J}^{T} R \mathfrak{J},
\end{aligned}
$$

where $\mathfrak{I}=\omega(b)+\omega(a)-2 / b-a \int_{a}^{b} \omega(u) d u$.

\section{Main Results}

Definition 1 (see [33]). The state $x(t)$ of the continuoustime system is GUUB, if for every $x(0) \in \mathbb{R}^{n}$, there exists a positive constant $\varepsilon$ and $a$ time $\bar{t}$ satisfying $x(t) \in\{x:\|x\| \leq \varepsilon\}, \forall \bar{t} \geq t$. Moreover, the continuous-time system is ultimately bounded or stable if its state $x(t)$ is GUUB

The objective of this section is to explore system (3) meeting the following requirements:

(i) System (3) is globally uniformly ultimately bounded (GUUB) stability and eventually exponentially converges to the bounded region by LMI. (ii) System (3) guarantees, under zero-initial conditions, $\|z(t)\|<\gamma\|\omega(t)\|_{2}$ for all nonzero $\omega \in l_{2}\left[\begin{array}{ll}0 & \infty\end{array}\right)$ and a given proper positive constant scalar $\gamma$.

Theorem 1. Consider the closed-loop system (3) and parameters driving mechanism (2) with $\delta, \beta, h_{1}>0, h_{2}>0$, $h_{m}>0$. Given a scalar $\gamma>0$, the close system (2) meets Ho application if there exist matrices $P>0, H_{1}>0, H_{2}>0$, $H_{3}>0, \Phi>0, R_{1}>0, R_{2}>0, R_{3}>0$, J and $U_{i}(i=1,2,3,4)$, matrices $Z$ and $S$, with appropriate dimensions such that

$$
\left[\begin{array}{cccc}
\Gamma_{11}+J & \Gamma_{12} & 0 & 0 \\
* & \Gamma_{22} & \Gamma_{23} & 0 \\
* & * & H e\left(-B_{1}^{T} B_{1} Z\right) & \Gamma_{34} \\
* & * & * & -J
\end{array}\right]<0
$$

where

$$
\begin{aligned}
& \Gamma_{11}=\left[\begin{array}{ccccccccc}
W_{11} & h_{1}\left(R_{1} A\right)^{T} & a_{2}\left(R_{2} A\right)^{T} & a_{3}\left(R_{3} A\right)^{T} & D_{1}^{T} & 6 R_{1} & 0 & 0 & 0 \\
* & -R_{1} & 0 & 0 & 0 & 0 & 0 & 0 & 0 \\
* & * & -R_{2} & 0 & 0 & 0 & 0 & 0 & 0 \\
* & * & * & -R_{3} & 0 & 0 & 0 & 0 & 0 \\
* & * & * & * & -I & 0 & 0 & 0 & 0 \\
* & * & * & * & * & -12 R_{1} & 0 & 0 & 0 \\
* & * & * & * & * & * & -12 R_{2} & 0 & 0 \\
* & * & * & * & * & * & * & -12 R_{2} & 0 \\
* & * & * & * & * & * & * & * & -12 R_{3}
\end{array}\right] \\
& \Gamma_{12}=\left[\begin{array}{cccccc}
-B_{1} S & P C_{1} & B_{1} S D_{2} & -2 R_{1} & 0 & 0 \\
-h_{1} B_{1} S & h_{1} R_{1} C_{1} & h_{1} B_{1} S D_{2} & 0 & 0 & 0 \\
-a_{2} B_{1} S & a_{2} R_{2} C_{1} & a_{2} B_{1} S D_{2} & 0 & 0 & 0 \\
-a_{3} B_{1} S & a_{3} R_{3} C_{1} & a_{3} B_{1} S D_{2} & 0 & 0 & 0 \\
-B_{2} S & C_{2} & B_{2} S D_{2} & 0 & 0 & 0 \\
0 & 0 & 0 & 6 R_{1}^{T} & 0 & 0 \\
0 & 0 & -F_{27}^{T} & 6 R_{2}^{T} & -F_{47}^{T} & 0 \\
0 & 0 & -F_{28}^{T} & -F_{38}^{T} & 6 R_{2}^{T} & 0 \\
0 & 0 & 0 & 0 & 6 R_{3}^{T} & 6 R_{3}^{T}
\end{array}\right], \\
& \Gamma_{22}=\left[\begin{array}{cccccc}
(\delta-1) \Phi & 0 & -\delta \Phi D_{2} & 0 & 0 & 0 \\
* & -\gamma^{2} I & 0 & 0 & 0 & 0 \\
* & * & W_{22} & -F_{23} & -F_{24} & 0 \\
* & * & * & W_{33} & -F_{34} & 0 \\
* & * & * & * & W_{44} & -2 R_{3} \\
* & * & * & * & * & W_{55}
\end{array}\right] \text {, } \\
& \Gamma_{23}=\left[\begin{array}{c}
-\left(B_{1}^{T} B_{1} S\right)^{T} \\
0 \\
\left(B_{1}^{T} B_{1} S D_{2}\right)^{T} \\
0 \\
0 \\
0
\end{array}\right] \text {, }
\end{aligned}
$$




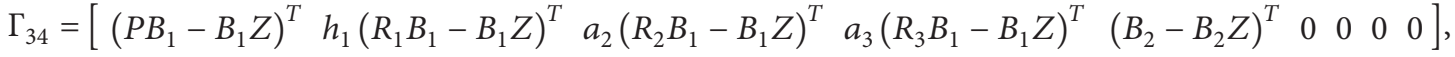

$$
\begin{aligned}
& \eta^{T}(t)=\left[\begin{array}{lllllllllll}
x^{T}(t) & x^{T}(t-\mathrm{d}(t)) & x^{T}\left(t-h_{1}\right) & x^{T}\left(t-h_{m}\right) & x^{T}\left(t-h_{2}\right) & Q_{1} & Q_{2} & Q_{3} & Q_{4} & e^{T}\left(i_{k} h\right) & \omega^{T}(t)
\end{array}\right], \\
& Q_{1}=\frac{1}{h_{1}} \int_{t-h_{1}}^{t} x^{T}(s) \mathrm{d} s, \\
& Q_{2}=\frac{1}{h_{1}-\mathrm{d}(t)} \int_{t-d(t)}^{t-h_{1}} x^{T}(s) \mathrm{d} s, \\
& Q_{3}=\frac{1}{h_{m}-d(t)} \int_{t-h_{m}}^{t-d(t)} x^{T}(s) \mathrm{d} s, \\
& Q_{4}=\frac{1}{h_{2}-h_{m}} \int_{t-h_{2}}^{t-h_{m}} x^{T}(s) \mathrm{d} s, \\
& W_{11}=H_{1}+H_{2}+H_{3}+\mathrm{He}(P A)-4 R_{1} \text {, } \\
& W_{22}=\delta D_{2}^{T} V D_{2}-8 R_{2}-\mathrm{He}\left(-U_{1}-U_{2}+U_{3}+U_{4}\right) \text {, } \\
& W_{33}=-H_{1}-4 R_{1}-4 R_{2} \text {, } \\
& W_{44}=-H_{2}-4 R_{2} \text {, } \\
& W_{55}=-H_{3}-4 R_{3} \text {, } \\
& F_{23}=2 R_{2}+U_{1}^{T}+U_{2}^{T}+U_{3}^{T}+U_{4}^{T} \text {, } \\
& F_{24}=2 R_{2}+U_{1}-U_{2}-U_{3}+U_{4} \text {, } \\
& F_{27}=-6 R_{2}-2\left(U_{3}^{T}+U_{4}^{T}\right) \text {, } \\
& F_{34}=-U_{1}+U_{2}-U_{3}+U_{4} \text {, } \\
& F_{38}=-2\left(U_{2}+U_{4}\right) \text {, } \\
& F_{47}=2\left(U_{3}^{T}-U_{4}^{T}\right) \text {. }
\end{aligned}
$$

Then, the closed-loop system (3) based on the mixed event trigger mechanism (2) achieves GUUB. When $t \longrightarrow \infty$, the system state $\xi(t)$ exponentially converges to the bounded area:

$$
\overline{\mathfrak{J}}=\left\{\begin{array}{l}
x(t) \in\left\{x:\|x(t)\| \leq \sqrt{\frac{\beta}{\delta \lambda_{\min (P)}}}\right\}, \quad \beta>0, \quad \alpha=0, \\
0, \quad \text { otherwise. }
\end{array}\right.
$$

Proof. Using the delay partitioning method to construct a suitable LKF for the paper,

$$
\begin{aligned}
V(t)= & x^{T}(t) P x(t)+\int_{t-h_{1}}^{t} x^{T}(s) H_{1} x(s) \mathrm{d} s+\int_{t-h_{m}}^{t} x^{T}(s) H_{2} x(s) \mathrm{d} s+\int_{t-h_{2}}^{t} x^{T}(s) H_{3} x(s) \mathrm{d} s \\
& +h_{1} \int_{-h_{1}}^{0} \int_{t+s}^{t} \dot{x}^{T}(v) R_{1} \dot{x}(v) \mathrm{d} v \mathrm{~d} s+a_{2} \int_{-h_{m}}^{-h_{1}} \int_{t+s}^{t} \dot{x}^{T}(v) R_{2} \dot{x}(v) \mathrm{d} v \mathrm{~d} s \\
& +a_{3} \int_{-h_{2}}^{-h_{m}} \int_{t+s}^{t} \dot{x}^{T}(v) R_{3} \dot{x}(v) \mathrm{d} v \mathrm{~d} s,
\end{aligned}
$$

where $a_{2}=d(t)-h_{1}$ and $a_{3}=h_{m}-d(t)$.

Taking the time derivative of (12) yields 


$$
\begin{aligned}
\dot{V}(t)= & 2 x^{T}(t) P x(t)+x^{T}(t)\left(H_{1}+H_{2}+H_{3}\right) \dot{x}(t)-x^{T}\left(t-h_{1}\right) H_{1} x\left(t-h_{1}\right)-x^{T}\left(t-h_{m}\right) H_{2} x\left(t-h_{m}\right), \\
& -x^{T}\left(t-h_{2}\right) H_{3} x\left(t-h_{2}\right)+h_{1}^{2} \dot{x}^{T}(t) R_{1} \dot{x}(t)+a_{2}^{2} \dot{x}^{T}(t) R_{2} \dot{x}(t)+a_{3}^{2} \dot{x}^{T}(t) R_{3} \dot{x}(t), \\
& -h_{1} \int_{t-h_{1}}^{t} \dot{x}^{T}(s) R \dot{x}(s) \mathrm{d} s-a_{2} \int_{t-h_{m}}^{t-h_{1}} \dot{x}^{T}(s) R \dot{x}(s) \mathrm{d} s-a_{3} \int_{t-h_{2}}^{t-h_{m}} \dot{x}^{T}(s) R \dot{x}(s) \mathrm{d} s+\rho^{T}\left(i_{k} h\right) \Phi \rho\left(i_{k} h\right) \\
& -\rho^{T}\left(i_{k} h\right) \Phi \rho\left(i_{k} h\right)+\gamma^{2} I \omega^{T}(t) \omega(t)-\gamma^{2} I \omega^{T}(t) \omega(t)-z^{T}(t) z(t)+z^{T}(t) z(t), \\
& \leq \eta^{T}(t)\left(\Xi_{1}-\Xi_{2}-\Xi_{3}+\bar{\Omega}^{T} R^{-1} \bar{\Omega}+\varsigma^{T} \varsigma\right) \eta(t)-z^{T}(t) z(t)+\gamma^{2} I \omega^{T}(t) \omega(t)+\vartheta(t) .
\end{aligned}
$$

$d(t) \in\left[h_{1}, h_{2}\right]$ can be divided into $d(t) \in\left[h_{1}, h_{m}\right]$ and $d(t) \in\left[h_{m}, h_{2}\right]$. We only discuss $d(t) \in\left[h_{1}, h_{m}\right]$ in this paper. The process of $d(t) \in\left[h_{m}, h_{2}\right]$ is similar to that of $h_{2}$ and $d(t) \in\left[h_{1}, h_{m}\right]$. Based on Lemma 3, $-\left(h_{m}-h_{1}\right) \int_{t-h_{m}}^{t-h_{1}} \dot{x}^{T}(s) R_{2} \dot{x}(s) \mathrm{d} s$ can be transformed as follows:

$$
\begin{aligned}
-\left(h_{m}-h_{1}\right) \int_{t-h_{m}}^{t-h_{1}} \dot{x}^{T}(s) R_{2} \dot{x}(s) \mathrm{d} s= & -\left(h_{m}-h_{1}\right) \int_{t-d(t)}^{t-h_{1}} \dot{x}^{T}(s) R_{2} \dot{x}(s) \mathrm{d} s-\left(h_{m}-h_{1}\right) \int_{t-h_{m}}^{t-d(t)} \dot{x}^{T}(s) R_{2} \dot{x}(s) \mathrm{d} s \\
& \leq-\frac{\left(h_{m}-h_{1}\right)}{\mathrm{d}(t)-h_{1}} \eta^{T}(t)\left[\begin{array}{ll}
e_{1}^{T} & e_{2}^{T}
\end{array}\right]\left[\begin{array}{ll}
R_{2} & 0 \\
0 & 3 R_{2}
\end{array}\right]\left[\begin{array}{l}
e_{1} \\
e_{2}
\end{array}\right] \eta(t) \\
& -\frac{\left(h_{m}-h_{1}\right)}{h_{m}-\mathrm{d}(t)} \eta^{T}(t)\left[\begin{array}{ll}
e_{3}^{T} & e_{4}^{T}
\end{array}\right]\left[\begin{array}{cc}
R_{2} & 0 \\
0 & 3 R_{2}
\end{array}\right]\left[\begin{array}{l}
e_{3} \\
e_{4}
\end{array}\right] \eta(t) \leq-\eta^{T}(t) \Xi_{2} \eta(t) .
\end{aligned}
$$

According to Lemma 3 , the integral term $-\left(h_{1}\right)$ $\int_{t-h_{1}}^{t} \dot{x}^{T}(s) R_{1} \dot{x}(s) \mathrm{d} s$ and $-\left(h_{2}-h_{m}\right) \int_{t-h_{2}}^{t-h_{m}} \dot{x}^{T}(s) R_{3} \dot{x}(s) \mathrm{d} s$ can be transformed as follows:

$$
\begin{aligned}
-h_{1} \int_{t-h_{1}}^{t} \dot{x}^{T}(s) R_{1} \dot{x}(s) \mathrm{d} s \leq & 4 x^{T}(t) R_{1} x(t)+4 x^{T}\left(t-h_{1}\right) R_{1} x(t)+2 x^{T}(t) R_{1} x\left(t-h_{1}\right)+2 x^{T}\left(t-h_{1}\right) R_{1} x(t) \\
& -\frac{6}{h_{1}} x^{T}(t) R_{1} \int_{t-h_{1}}^{t} \dot{x}(s) \mathrm{d} s-\frac{6}{h_{1}} \int_{t-h_{1}}^{t} \dot{x}^{T}(s) \mathrm{d} s R_{1} x(t)-\frac{6}{h_{1}} x^{T}\left(t-h_{1}\right) R_{1} t \int_{t-h_{1}}^{t} \dot{x}(s) \mathrm{d} s \\
& -\frac{6}{h_{1}} \int_{t-h_{1}}^{t} \dot{x}^{T}(s) \mathrm{d} s R_{1} x\left(t-h_{1}\right)-a_{3} \int_{t-h_{2}}^{t-h_{m}} \dot{x}^{T}(s) R_{3} \dot{x}(s) \mathrm{d} s \\
\leq & 4 x^{T}\left(t-h_{m}\right) R_{3} x\left(t-h_{m}\right)+4 x^{T}\left(t-h_{2}\right) R_{3} x\left(t-h_{2}\right)+2 x^{T}\left(t-h_{m}\right) R_{3} x\left(t-h_{2}\right) \\
& +2 x^{T}\left(t-h_{2}\right) R_{3} x\left(t-h_{m}\right)-\frac{6}{a_{3}} x^{T}\left(t-h_{m}\right) R_{3} \int_{t-h_{2}}^{t-h_{m}} \dot{x}(s) \mathrm{d} s-\frac{6}{a_{3}} \int_{t-h_{2}}^{t-h_{m}} \dot{x}^{T}(s) \mathrm{d} s R_{3} x\left(t-h_{m}\right) \\
& -\frac{6}{a_{3}} x^{T}\left(t-h_{2}\right) R_{3} \int_{t-h_{2}}^{t-h_{m}} \dot{x}(s) \mathrm{d} s-\frac{6}{a_{3}} \int_{t-h_{2}}^{t-h_{m}} \dot{x}^{T}(s) \mathrm{d} s R_{3} x\left(t-h_{2}\right) .
\end{aligned}
$$

So, $-h_{1} \int_{t-h_{1}}^{t} \dot{x}^{T}(s) R_{1} \dot{x}(s) \mathrm{d} s-h_{1} \int_{t-h_{1}}^{t} \dot{x}^{T}(s) R_{1} \dot{x} \quad(s) \mathrm{d} s$ $\leq-\eta^{T}(t) \Xi_{3} \eta(t)$, where $Q_{11}=H_{1}+H_{2}+H_{3}+\operatorname{He}(P A)$ : 


$$
\begin{aligned}
& \Xi_{1}=\left[\begin{array}{ccccccccccc}
Q_{11} & P B_{1} K D_{2} & 0 & 0 & 0 & 0 & 0 & 0 & 0 & -P B_{1} K & P C_{1} \\
* & \delta D_{2}^{T} \Phi D_{2} & 0 & 0 & 0 & 0 & 0 & 0 & 0 & -\delta D_{2}^{T} V & 0 \\
* & * & -H_{1} & 0 & 0 & 0 & 0 & 0 & 0 & 0 & 0 \\
* & * & * & -H_{2} & 0 & 0 & 0 & 0 & 0 & 0 & 0 \\
* & * & * & * & -H_{3} & 0 & 0 & 0 & 0 & 0 & 0 \\
* & * & * & * & * & -12 R_{1} & 0 & 0 & 0 & 0 & 0 \\
* & * & * & * & * & * & * & -12 R_{2} & 0 & 0 & 0 \\
* & * & * & * & * & * & * & * & -12 R_{3} & 0 & 0 \\
* & * & * & * & * & * & * & * & * & (\delta-1) \Phi & 0 \\
* & * & * & * & * & * & * & * & * & * & -\gamma^{2} I
\end{array}\right] \text {, } \\
& \Xi_{2}=\left[\begin{array}{ccccccccccc}
0 & 0 & 0 & 0 & 0 & 0 & 0 & 0 & 0 & 0 & 0 \\
* & F_{22} & F_{23} & F_{24} & 0 & 0 & F_{27} & F_{28} & 0 & 0 & 0 \\
* & * & 4 R_{2} & F_{34} & 0 & 0 & -6 R_{2} & F_{38} & 0 & 0 & 0 \\
* & * & * & 4 R_{2} & 0 & 0 & F_{47} & -6 R_{2} & 0 & 0 & 0 \\
* & * & * & * & 0 & 0 & 0 & 0 & 0 & 0 & 0 \\
* & * & * & * & * & 0 & 0 & 0 & 0 & 0 & 0 \\
* & * & * & * & * & * & * & 12 R_{2} & 0 & 0 & 0 \\
* & * & * & * & * & * & * & * & 0 & 0 & 0 \\
* & * & * & * & * & * & * & * & * & 0 & 0 \\
* & * & * & * & * & * & * & * & * & * & 0
\end{array}\right],
\end{aligned}
$$

$$
\Xi_{3}=\left[\begin{array}{ccccccccccc}
4 R_{1} & 0 & 2 R_{1} & 0 & 0 & -6 R_{1} & 0 & 0 & 0 & 0 & 0 \\
* & 0 & 0 & 0 & 0 & 0 & 0 & 0 & 0 & 0 & 0 \\
* & * & 4 R_{1} & 0 & 0 & -6 R_{1} & 0 & 0 & 0 & 0 & 0 \\
* & * & * & 4 R_{3} & 2 R_{3} & 0 & 0 & 0 & -6 R_{3} & 0 & 0 \\
* & * & * & * & 4 R_{3} & 0 & 0 & 0 & -6 R_{3} & 0 & 0 \\
* & * & * & * & * & 12 R_{1} & 0 & 0 & 0 & 0 & 0 \\
* & * & * & * & * & * & 0 & 0 & 0 & 0 & 0 \\
* & * & * & * & * & * & * & 0 & 0 & 0 & 0 \\
* & * & * & * & * & * & * & * & 12 R_{3} & 0 & 0 \\
* & * & * & * & * & * & * & * & * & 0 & 0 \\
* & * & * & * & * & * & * & * & * & * & 0
\end{array}\right],
$$

$\bar{\Omega}_{1}=\left[\begin{array}{lllllllllll}h_{1} R_{1} A & h_{1} R_{1} B_{1} K D_{2} & 0 & 0 & 0 & 0 & 0 & 0 & 0 & -h_{1} R_{1} B_{1} K & h_{1} R_{1} C_{1}\end{array}\right]$,

$\bar{\Omega}_{2}=\left[\begin{array}{lllllllllll}a_{2} R_{2} A & a_{2} R_{2} B_{1} K D_{2} & 0 & 0 & 0 & 0 & 0 & 0 & 0 & -a_{2} R_{2} B_{1} K & a_{2} R_{2} C_{1}\end{array}\right]$,

$\bar{\Omega}_{3}=\left[\begin{array}{lllllllllll}a_{3} R_{3} A & a_{3} R_{3} B_{1} K D_{2} & 0 & 0 & 0 & 0 & 0 & 0 & 0 & -a_{3} R_{3} B_{1} K & a_{2} R_{3} C_{1}\end{array}\right]$,

$$
\varsigma=\left[\begin{array}{lllllllllllll}
D_{1}^{T} & B_{2} K D_{2} & 0 & 0 & 0 & 0 & 0 & 0 & 0 & -B_{2} K & C_{2}
\end{array}\right] \text {. }
$$


To make closed-loop system (3) meet the Ho appli- $\quad$ Through Schur supplement for (18), we can obtain cations, we can conclude

$$
\begin{array}{r}
\Xi_{1}-\Xi_{2}-\Xi_{3}+\bar{\Omega}_{1}^{T} R_{1}^{-1} \bar{\Omega}_{1}+\bar{\Omega}_{2}^{T} R_{2}^{-1} \bar{\Omega}_{2}+\bar{\Omega}_{3}^{T} R_{3}^{-1} \bar{\Omega}_{3}+\varsigma^{T}, \\
\varsigma<0 .
\end{array}
$$

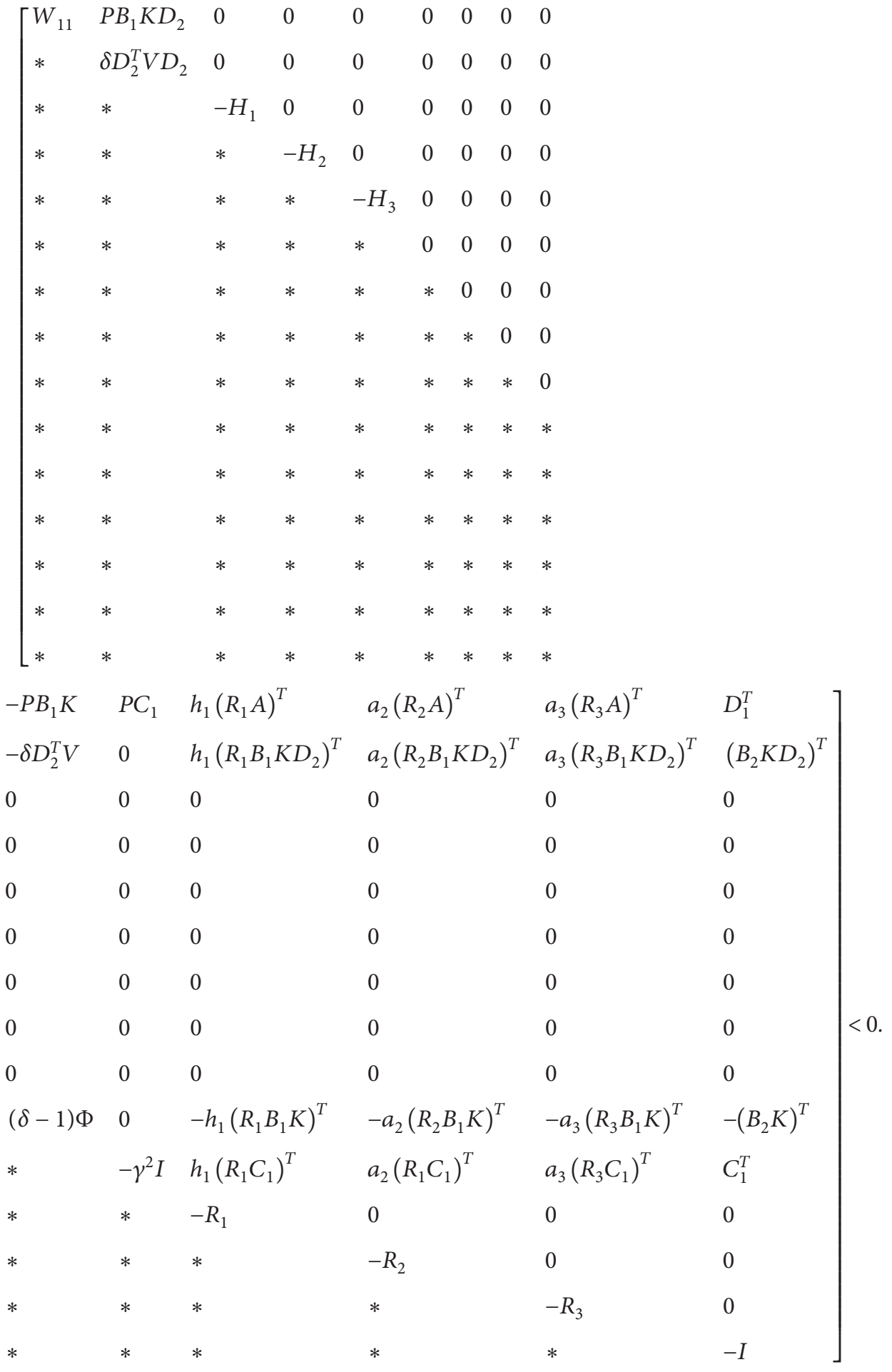


Multiplying left by $\Lambda$ and right by $\Lambda^{\mathrm{T}}$, we have

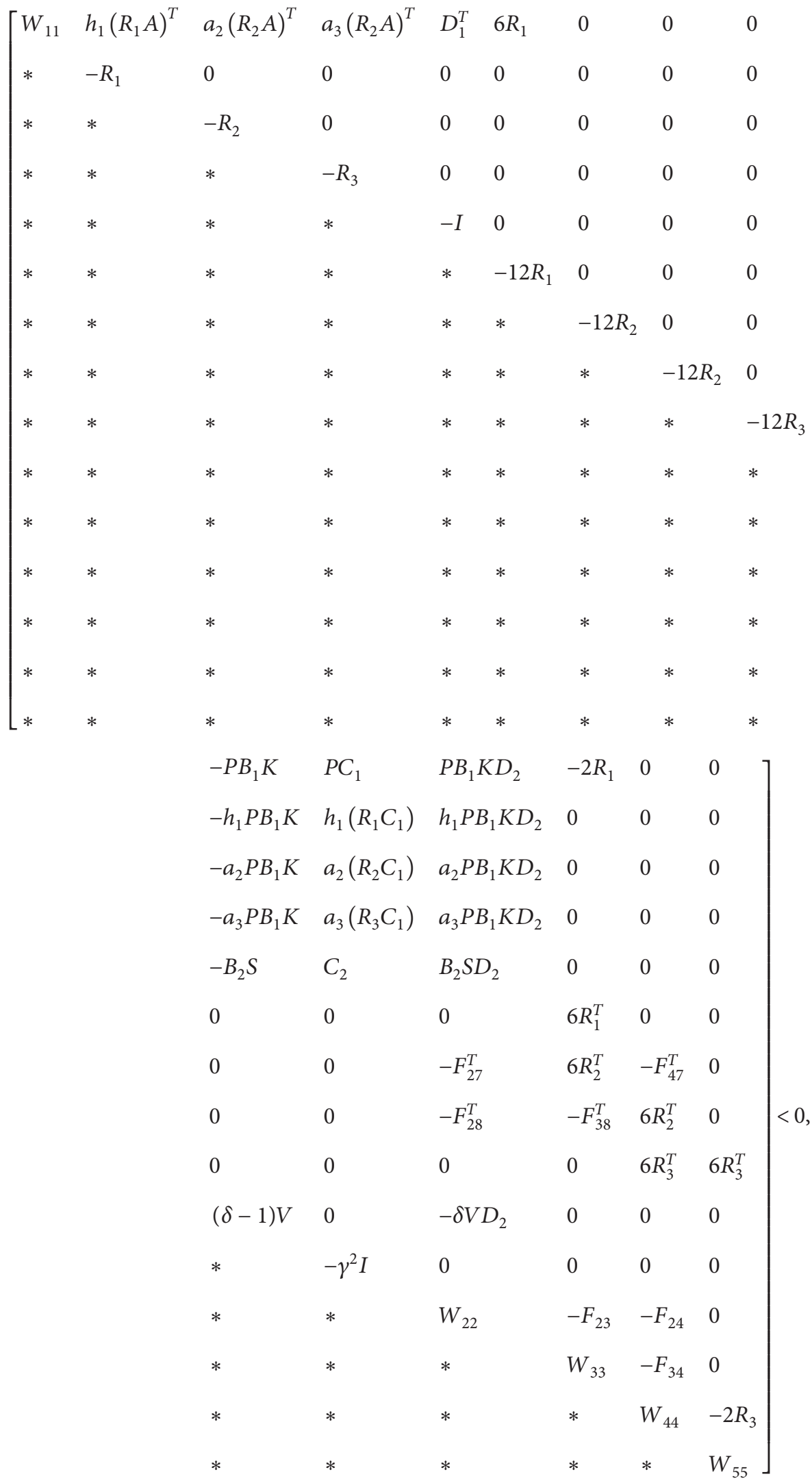


where

$$
\Lambda=\left[\begin{array}{lllllllllllllll}
I & 0 & 0 & 0 & 0 & 0 & 0 & 0 & 0 & 0 & 0 & 0 & 0 & 0 & 0 \\
0 & 0 & 0 & 0 & 0 & 0 & 0 & 0 & 0 & 0 & 0 & I & 0 & 0 & 0 \\
0 & 0 & 0 & 0 & 0 & 0 & 0 & 0 & 0 & 0 & 0 & 0 & I & 0 & 0 \\
0 & 0 & 0 & 0 & 0 & 0 & 0 & 0 & 0 & 0 & 0 & 0 & 0 & I & 0 \\
0 & 0 & 0 & 0 & 0 & 0 & 0 & 0 & 0 & 0 & 0 & 0 & 0 & 0 & I \\
0 & 0 & 0 & 0 & 0 & I & 0 & 0 & 0 & 0 & 0 & 0 & 0 & 0 & 0 \\
0 & 0 & 0 & 0 & 0 & 0 & I & 0 & 0 & 0 & 0 & 0 & 0 & 0 & 0 \\
0 & 0 & 0 & 0 & 0 & 0 & 0 & I & 0 & 0 & 0 & 0 & 0 & 0 & 0 \\
0 & 0 & 0 & 0 & 0 & 0 & 0 & 0 & I & 0 & 0 & 0 & 0 & 0 & 0 \\
0 & 0 & 0 & 0 & 0 & 0 & 0 & 0 & 0 & I & 0 & 0 & 0 & 0 & 0 \\
0 & I & 0 & 0 & 0 & 0 & 0 & 0 & 0 & 0 & 0 & 0 & 0 & 0 & 0 \\
0 & 0 & I & 0 & 0 & 0 & 0 & 0 & 0 & 0 & 0 & 0 & 0 & 0 & 0 \\
0 & 0 & 0 & I & 0 & 0 & 0 & 0 & 0 & 0 & 0 & 0 & 0 & 0 & 0 \\
0 & 0 & 0 & 0 & I & 0 & 0 & 0 & 0 & 0 & 0 & 0 & 0 & 0 & 0
\end{array}\right] .
$$

Let $K=Z^{-1} S$, and (18) can be rewritten as follows:

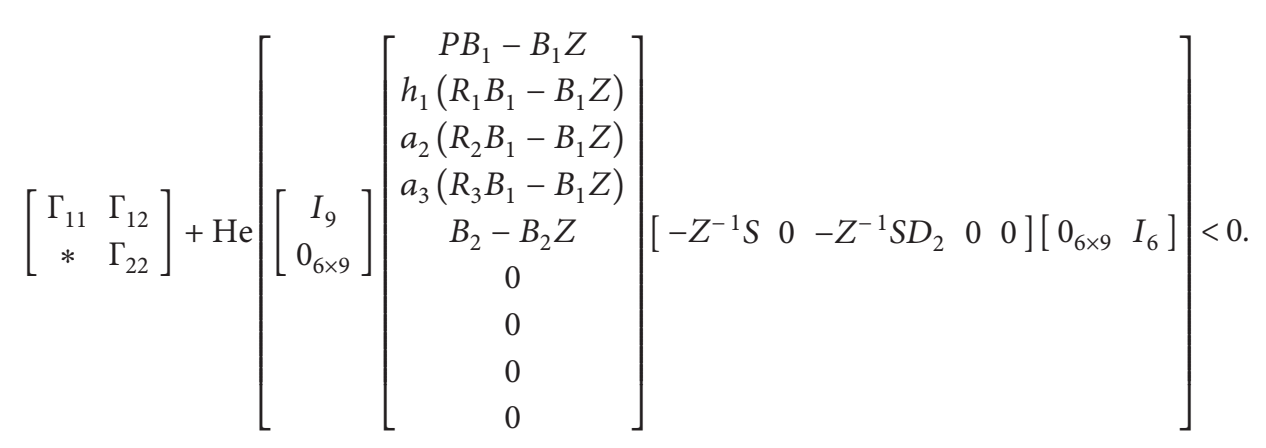

Applying Lemma 1, we can obtain

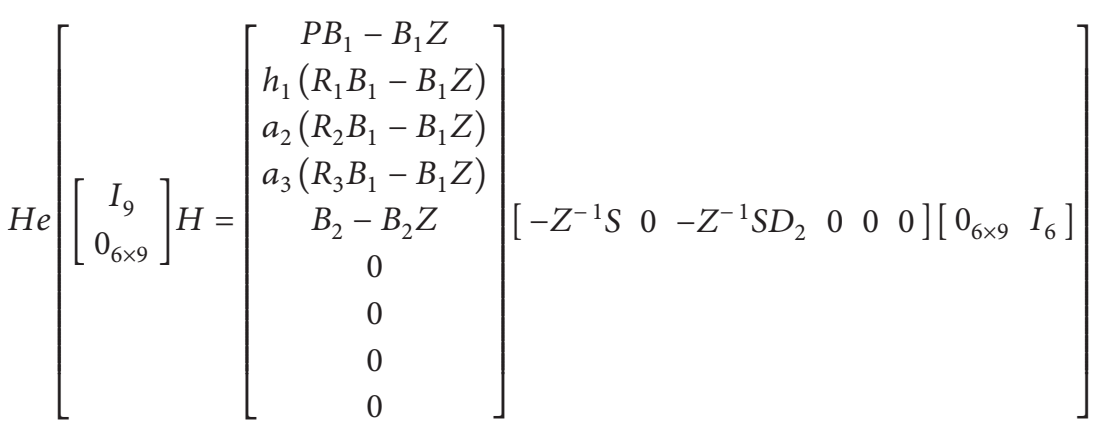

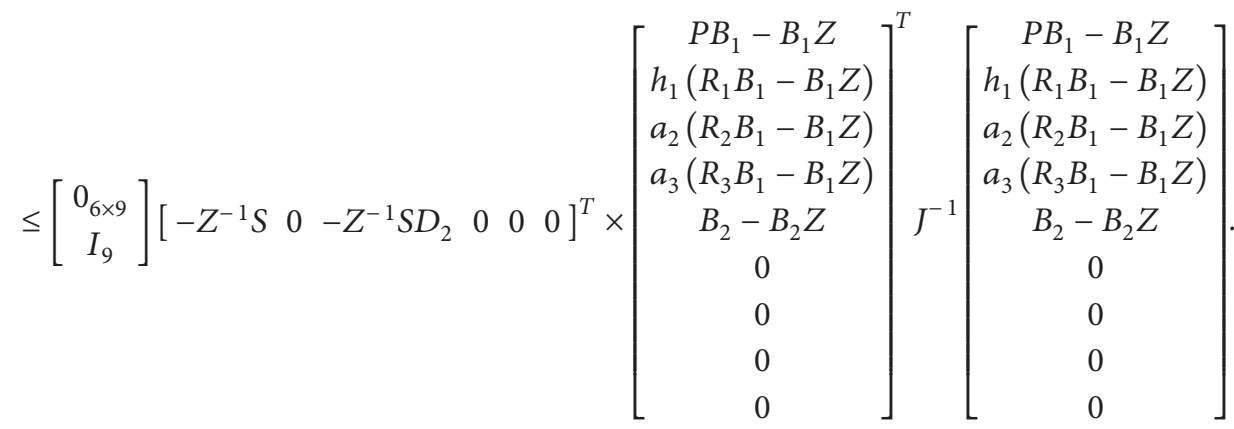


Combining (20) and (22) can lead to

$$
\left[\begin{array}{ccc}
\Gamma_{11} & \Gamma_{12} & 0 \\
* & \Gamma_{22} & E^{T} \\
* & * & H^{T} J^{-1} H
\end{array}\right]<0,
$$

where

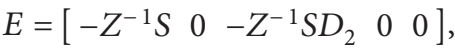

$$
\begin{aligned}
& H=\left[\begin{array}{c}
P B_{1}-B_{1} Z \\
\sqrt{h_{1}}\left(R_{1} B_{1}-B_{1} Z\right) \\
\sqrt{a_{2}}\left(R_{2} B_{1}-B_{1} Z\right) \\
\sqrt{a_{3}}\left(R_{3} B_{1}-B_{1} Z\right) \\
B_{2}-B_{2} Z \\
0 \\
0 \\
0 \\
0
\end{array}\right] .
\end{aligned}
$$

Through Lemma 2 and Schur complement to (24), we can obtain

$$
\left[\begin{array}{cccc}
\Gamma_{11}+J & \Gamma_{12} & 0 & 0 \\
* & \Gamma_{22} & E^{T} G^{T} B_{1}^{T} B_{1} & 0 \\
* & * & H e\left(-B_{1}^{T} B_{1} G\right) & \Gamma_{34} \\
* & * & * & -J
\end{array}\right]<0 .
$$

So, we can obtain that

$$
\begin{array}{r}
\eta^{T}(t)\left(\Xi_{1}-\Xi_{2}-\Xi_{3}+\bar{\Omega}_{1}^{T} R_{1}^{-1} \bar{\Omega}_{1}+\bar{\Omega}_{2}^{T} R_{2}^{-1} \bar{\Omega}_{2}+\bar{\Omega}_{3}^{T} R_{3}^{-1} \bar{\Omega}_{3}+\varsigma^{T} \varsigma\right), \\
\eta(t)<0,
\end{array}
$$

and we can conclude

$$
\begin{aligned}
\dot{V}(t)+\delta V(t) \leq & \eta^{T}(t)\left(\Xi_{1}-\Xi_{2}-\Xi_{3}+\bar{\Omega}_{1}^{T} R_{1}^{-1} \bar{\Omega}_{1}+\bar{\Omega}_{2}^{T} R_{2}^{-1} \bar{\Omega}_{2}\right. \\
& \left.+\bar{\Omega}_{3}^{T} R_{3}^{-1} \bar{\Omega}_{3}+\varsigma^{T} \varsigma\right) \eta(t) \eta(t)-z^{T}(t) z(t) \\
& +\gamma^{2}\|\omega(t)\|_{2}+\vartheta(t) \leq \gamma^{2}\|\omega(t)\|_{2}+\vartheta(t) .
\end{aligned}
$$

Applying comparison lemma [34] to (26),

$$
V(t) \leq e^{-\delta} V(0)+\psi(t) \frac{\gamma}{\delta}\left(1-e^{\delta t}\right)\|\omega(t)\|_{2},
$$

where $\psi(t)=\int_{0}^{t} e^{-\delta(t-s)}\left(\beta \varepsilon^{-\alpha s}\right) \mathrm{d} s$.

We consider the following situations by category.

(i) If $\beta=0$, we can conclude $\psi(t)=0$ :

$$
V(t) \leq e^{-\delta}\left(V(0)-\frac{\gamma}{\delta}\|\omega(t)\|_{2}\right)+\frac{\gamma}{\delta}\|\omega(t)\|_{2} .
$$

(ii) If $\beta>0, \alpha=0$, we can conclude $\psi(t)=\gamma / \delta\left(1-e^{\delta t}\right)$ :

$$
V(t) \leq e^{-\delta}\left(V(0)-\frac{\gamma}{\delta}\|\omega(t)\|_{2}-\frac{\gamma}{\delta}\right)+\frac{\gamma}{\delta}\|\omega(t)\|_{2}+\frac{\gamma}{\delta} .
$$

(iii) If $\beta>0, \alpha>0, \delta-\alpha \ln \varepsilon=0$, we can conclude $\psi(t)=\beta t e^{-\alpha t}$ :

$$
V(t) \leq e^{-\delta}\left(V(0)-\frac{\gamma}{\delta}\|\omega(t)\|_{2}+\beta t\right)+\frac{\gamma}{\delta}\|\omega(t)\|_{2} .
$$

(iv) If $\beta>0, \alpha>0, \delta-\alpha \ln \varepsilon>0$, we can conclude $\psi(t)=\beta / \delta-\alpha \ln \varepsilon\left(\varepsilon^{-\alpha t}-e^{-\delta t}\right)$ :

$$
\begin{aligned}
V(t) \leq & e^{-\delta}\left(V(0)-\frac{\gamma}{\delta}\|\omega(t)\|_{2}+\beta t-\frac{\beta}{\delta-\alpha \ln \varepsilon}\right)+\frac{\gamma}{\delta}\|\omega(t)\|_{2} \\
& +\frac{\beta}{\delta-\alpha \ln \varepsilon} \varepsilon^{-\alpha t} .
\end{aligned}
$$

Define $\psi_{\max }=\max \psi(t)$ and union (30)-(33) can lead:

$$
\psi_{\max }= \begin{cases}0, & \beta=0, \\ \frac{\beta}{\delta}, & \beta>0, \alpha>0, \\ \frac{\beta \varepsilon^{\alpha h_{2}}}{\delta e}, & \beta>0, \delta-\alpha \ln \varepsilon=0, \\ \frac{\beta \varepsilon^{\alpha h_{2}}}{|\delta-\alpha \ln \varepsilon|}, & \beta>0, \alpha>0, \delta-\alpha \ln \varepsilon \neq 0 .\end{cases}
$$

Accordingly, LKF can lead $x^{T}(t) P x(t) \leq V(t)$. If $\omega(t)=0$, we can obtain

$$
x^{T}(t) P x(t) \leq V(t) \leq V(0)+\psi_{\max }
$$

and we delimit

$$
\mathfrak{I}=\sqrt{\frac{V(0) \psi_{\max }}{\lambda_{\text {min }}}(P) .}
$$

Accordingly, (34) and (35) can lead:

$$
x(t) \in\{x:\|x(t)\| \leq \mathfrak{J}\}, \quad t>0 .
$$

Meanwhile, if $\omega(t)=0$, accordingly, (36) can lead:

$$
\lim _{t \rightarrow \infty} V(t) \leq \begin{cases}\frac{\beta}{\delta}, & \beta>0, \alpha=0, \\ 0, & \text { otherwise, }\end{cases}
$$

when $t \longrightarrow \infty$, (12) exponentially converges to the bounded region: 


$$
\overline{\mathfrak{I}}= \begin{cases}x(t) \in\left\{x:\|x(t)\| \leq \sqrt{\left.\frac{\beta}{\delta \lambda_{\min (P)}}\right\},}\right. & \beta>0, \alpha=0, \\ 0, & \text { otherwise. }\end{cases}
$$

Then, we can get the closed-loop system (3) is GUUB when $t \longrightarrow \infty, \omega(t)=0$ the system state exponentially converges to the bounded area. At the same time, $\|z(t)\|<\gamma\|\omega(t)\|_{2}$ at the zero-initial conditions is obtained.

Remark 1. Compared with [15], this paper has three different characteristics.(1) A mixed event triggering mechanism, in which absolute trigger mechanism is added to the relative to the trigger term. (2) The concept of the time-delay partitions was applied to build a LKF. (3) The external disturbance is added to the original system and $H \infty$ performance index can be obtained. The purpose of the three improved views is to make the system robust.

Remark 2. Another class of method is reducing the conservation of simple LKF. The augmented LKF is established,
TABle 1: The controller gain $K$ and the weight matrix $\Phi$.

\begin{tabular}{lccc}
\hline$\delta$ & 0.1 & 0.2 & 0.3 \\
$K$ & -0.0684 & -0.0130 & -0.0114 \\
$\Phi$ & 163 & 58.7500 & 27.7632 \\
\hline
\end{tabular}

in which it contains not only the state vector but also the derivative of the state. Meanwhile, the free-weight matrix inequality is raised in the study of integral inequalities. The free-weight matrix inequality adds some freedoms to the system based on the Wirtinger inequality. Constructing a suitable LKF by combining the two methods above, the function can be selected as

$$
\begin{aligned}
V(t)= & \xi_{1}^{T}(t) P \xi_{1}(t)+\int_{t-h_{1}}^{t} \xi_{2}^{T}(s) H_{1} \xi_{2}(s) \mathrm{d} s \\
& +\int_{t-h_{2}}^{t} \xi_{2}^{T}(s) H_{2} \xi_{2}(s) \mathrm{d} s \\
& +\int_{-h_{1}}^{0} \int_{t+s}^{t} \dot{x}^{T}(v) R_{1} \dot{x}(v) \mathrm{d} v \mathrm{~d} s \\
& +\int_{-h_{2}}^{-h_{1}} \int_{t+s}^{t} \dot{x}^{T}(v) R_{2} \dot{x}(v) \mathrm{d} v \mathrm{~d} s,
\end{aligned}
$$

where

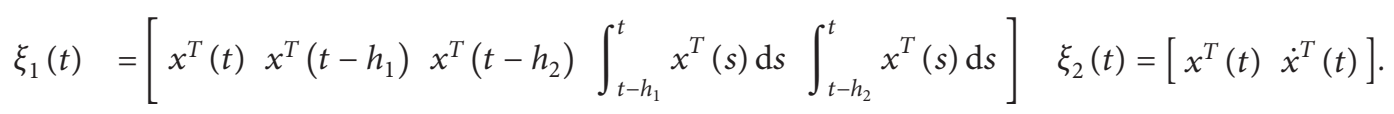

\section{Simulation}

This section provides two numerical simulation examples. The first example shows that the proposed method is effective. The second example shows the dynamic response of the system in the event-driven mode.

Example 1. Given the following parameters,

$$
\begin{aligned}
A & =\left[\begin{array}{ll}
-0.2 & -0.1 \\
0.2 & -0.5
\end{array}\right], \\
B_{1} & =\left[\begin{array}{l}
1 \\
1
\end{array}\right], \\
C_{1} & =\left[\begin{array}{ll}
0 & 1
\end{array}\right], \\
D_{1} & =\left[\begin{array}{ll}
0.3 & 1
\end{array}\right], \\
D_{2} & =\left[\begin{array}{ll}
1 & 0
\end{array}\right], \\
B_{2} & =0.1 .
\end{aligned}
$$

Apply Theorem 1 with $h=0.01 \mathrm{~s}, d_{M}=0.5$, and $\gamma=50$. With the change of $\delta$, the corresponding controller gain $K$ and event-driven matrix $\Phi$ are listed in Table 1.

$$
\delta=0.2, \alpha_{1}=0, \beta=0.1, \alpha=0.5, \varepsilon=e, \text { and } \varepsilon_{0}=0.01
$$

Example 2. Given $\delta=0.1, \quad h_{1}=0.01, h_{2}=0.15, \quad h_{m}=0.1$, controller gain $K=-0.00684$, weight matrix $\Phi=2921.3$, $h=0.01$, and $\gamma=50$, according to the above conditions, system (1) is stable in the event-driven mode. $x$ initial conditions are $x(0)=\left[\begin{array}{c}1 \\ -1\end{array}\right], \omega(t)=0.09 * \sin 0.2 * t$.

If $\delta=0.1, \beta=0$ result is shown in following figures. According to the above set parameters in the paper, some simulation results are shown in Figure 1.

From Figure 1, we can find that the system actually works under the relative trigger mechanism. When the output error reaches a certain threshold, the system state updates and then presents a periodic update state. The length of update interval will depend on the setting of $\delta$ value.

If $\delta=0, \beta=0.3$ result is shown in Figure 2 .

From Figure 2, we can find that the actual operation of the system is under the absolute trigger mechanism, and the trigger threshold is independent of the system state. Depending on the setting of $\beta$ value, it is obvious that the update time of the system is earlier and the update interval is denser.

If $\delta=0.1, \beta=0.3$ result is shown in Figure 3 .

From Figure 3, we can find that the system actually works under the mixed trigger mechanism. The trigger 


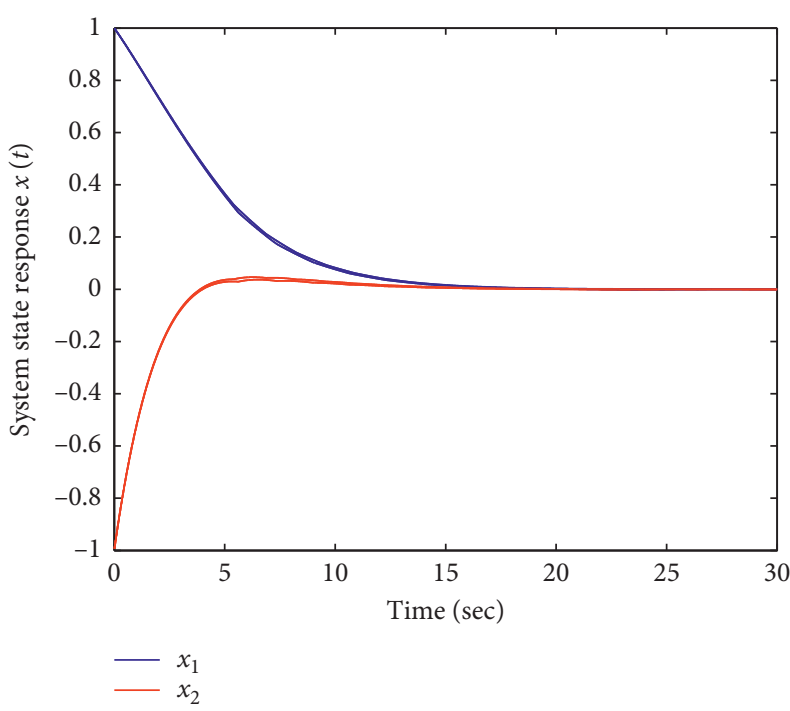

(a)

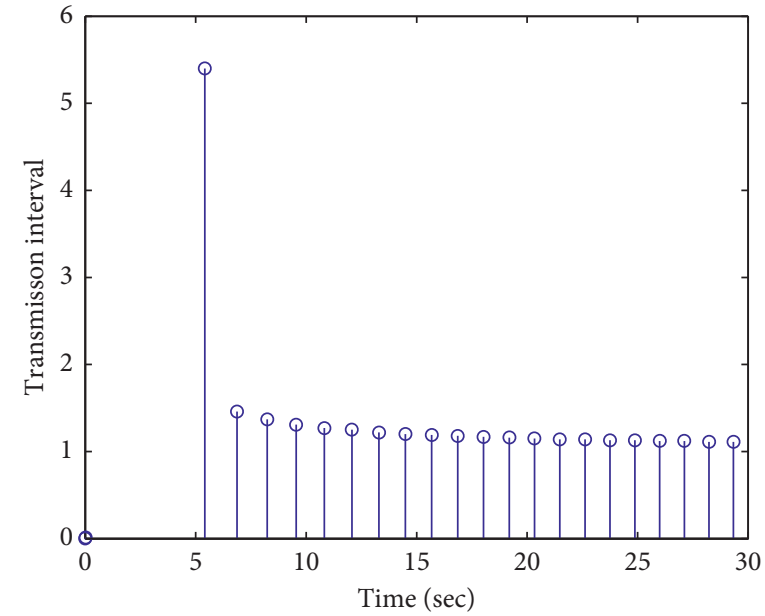

(b)

FIGURE 1: System state responses diagram and event-driven update picture. (a) Parameters' change of $x(t)$. (b) Event-driven updates.

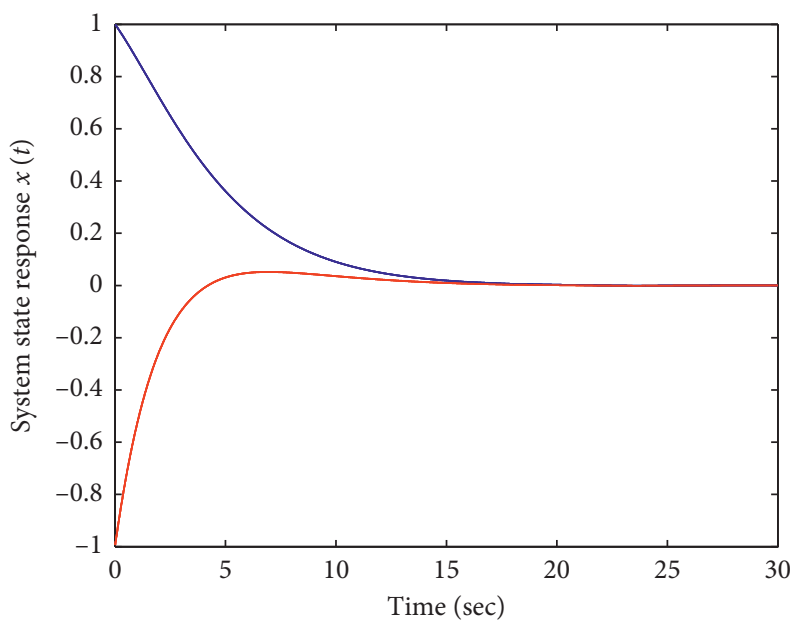

(a)

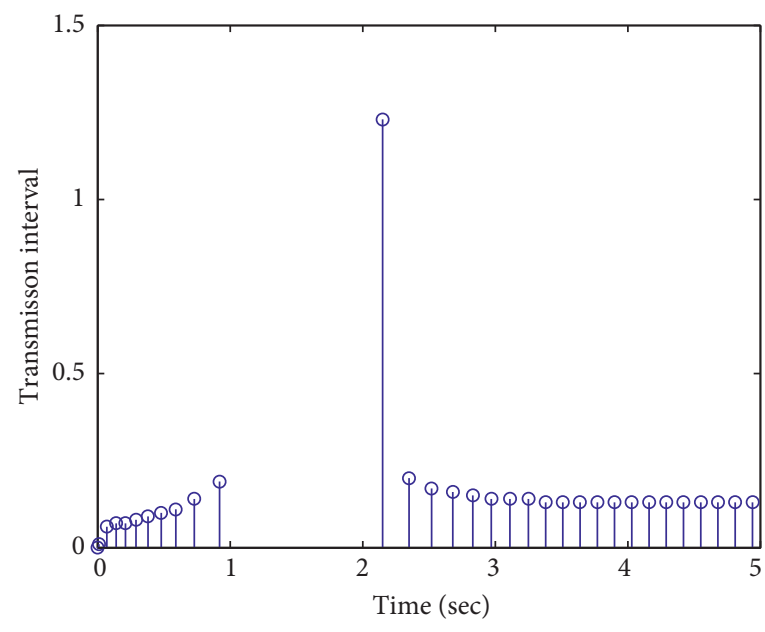

(b)

Figure 2: System state response diagram and event-driven update picture. (a) Parameters' change of $x(t)$. (b) Event-driven updates.

threshold is related not only to the system state but also to the initially set $\beta$ value. We can see that the number of system updates is significantly reduced and the update interval is longer.

Through the above simulation, it can be found that different trigger mechanisms can stabilize the system, but the update time of the system state is obviously different. Among them, the hybrid trigger mechanism proposed in this paper has more effective results, which can greatly reduce the network burden and save network bandwidth compared with the other two trigger methods. 


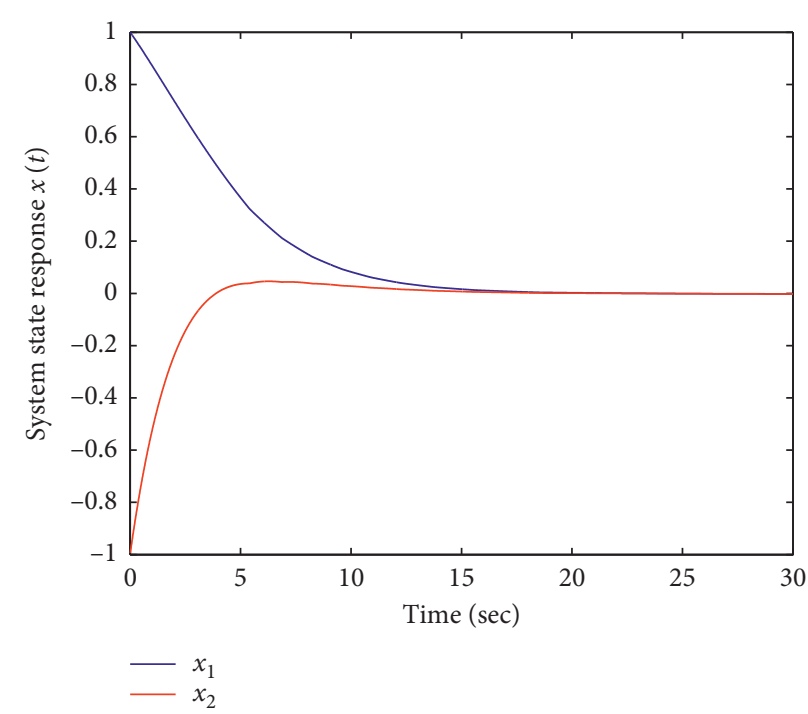

(a)

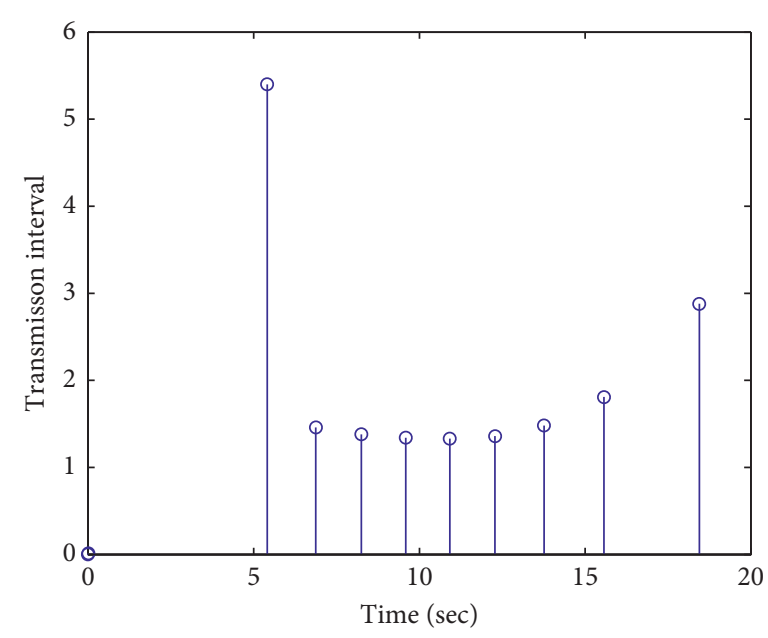

(b)

FIgURe 3: System state response diagram and event-driven update picture. (a) Parameters' change of $x(t)$. (b) Event-driven updates.

\section{Conclusion}

This paper has investigated the event-driven problem about NCSs. A mixed event trigger mechanism is introduced, which contains absolute trigger mechanism and relative trigger. The event triggering mechanism can be expressed by a time-delay model. The coupling of the system is reduced by introducing additional parameters and matrices. The sufficient condition can be obtained by LMI. By using the Wirtinger inequality method, relatively event-driven controller can be obtained, which meets an Ho performance index level of NCSs. Finally, two numerical simulation examples illustrate the effectiveness of the proposed method. In networked control systems, besides time delay, packet loss and quantization are also important factors that affect system performance. How to comprehensively consider the above two factors combined with trigger mechanism will be the focus of future work.

\section{Data Availability}

The data used to support the findings of this study are included within the article. Because it is a numerical simulation example, readers can get the same results as this article by using the LMI toolbox of Matlab and the theorem given in this article.

\section{Conflicts of Interest}

The authors declare that they have no conflicts of interest.

\section{Acknowledgments}

This work was supported by National Natural Science Foundation of China (Grant nos. 61403278 and 61503280).

\section{References}

[1] X. M. Zhang, Q. L. Han, and X. H. Ge, "Networked control systems: a survey of trends and techniques," IEEE-CAA Journal of Automatica Sinica, vol. 7, no. 1, pp. 1-17, 2020.

[2] H. Yan, C. Hu, H. Zhang, H. R. Karimi, X. Jiang, and M. Liu, “\$H_\{ $\$$ infty\}\$ output tracking control for networked systems with adaptively adjusted event-triggered scheme," IEEE Transactions on Systems, Man, and Cybernetics: Systems, vol. 49, no. 10, pp. 2050-2058, 2019.

[3] N. Xu and L. Sun, "An improved delay-dependent stability analysis for markovian jump systems with interval timevarying-delays," IEEE Access, vol. 6, pp. 33055-33061, 2018.

[4] N. Xu and L. Sun, "Synchronization control of markov jump neural networks with mixed time-varying delay and parameter uncertain based on sample point controller," Nonlinear Dynamics, vol. 98, no. 3, pp. 1877-1890, 2019.

[5] Z. Li, H. Yan, H. Zhang, X. Zhan, and C. Huang, "Stability analysis for delayed neural networks via improved auxiliary polynomial-based functions," IEEE Transactions on Neural Networks and Learning Systems, vol. 30, no. 8, pp. 2562-2568, 2019.

[6] X. Li, B. Zhang, P. Li et al., "Finite-horizon $\$ \mathrm{H}_{-}\{$infty\}\$ state estimation for periodic neural networks over fading channels," IEEE Transactions on Neural Networks and Learning Systems, vol. 31, no. 5, pp. 1450-1460, 2020.

[7] G. Nagamani, Y. H. Joo, and T. Radhika, "Delay-dependent dissipativity criteria for markovian jump neural networks with random delays and incomplete transition probabilities," Nonlinear Dynamics, vol. 91, no. 56, pp. 2503-2522, 2018.

[8] X.-M. Li, Q. Zhou, P. Li, H. Li, and R. Lu, "Event-triggered consensus control for multi-agent systems against false datainjection attacks," IEEE Transactions on Cybernetics, vol. 50, no. 5, pp. 1856-1866, 2020.

[9] M. S. Ali and R. Saravanakumar, "Robust h-infinity control of uncertain systems with two additive time-varying delays," Chinese Physics B, vol. 24, no. 9, pp. 90202-90211, 2015.

[10] R. Saravanakumar, M. S. Ali, and H. R. Karimi, "Robust hoo control of uncertain stochastic markovian jump systems with 
mixed time-varying delays," International Journal of Systems Science, vol. 48, no. 4, pp. 862-872, 2017.

[11] M. Shen, J. H. Park, and D. Ye, "A separated approach to control of markov jump nonlinear systems with general transition probabilities," IEEE Transactions on Cybernetics, vol. 46, no. 9, pp. 2010-2018, 2016.

[12] M. Shen, S. Yan, and G. M. Zhang, "A new approach to eventtriggered static output feedback control of networked control systems," ISA Transactions, vol. 65, pp. 468-474, 2016.

[13] C. Peng and Q.-L. Han, "On designing a novel self-triggered sampling scheme for networked control systems with data losses and communication delays," IEEE Transactions on Industrial Electronics, vol. 63, no. 2, pp. 1239-1248, 2016.

[14] Y. Shen and M. Q. Shen, "Guangming zhanga: "extended event-driven observer-based output control of networked control systems," Nonlinear Dynamics, vol. 86, no. 17, pp. 1639-1648, 2016.

[15] H. Zhang, Z. Wang, H. Yan, F. Yang, and X. Zhou, “Adaptive event-triggered transmission scheme and $\$ \mathrm{~h} \_\{\backslash \text { infty }\} \$$ filtering co-design over a filtering network with switching topology," IEEE Transactions on Cybernetics, vol. 49, no. 12, pp. 4296-4307, 2019.

[16] Z. Gu, P. Shi, and D. Yue, "An adaptive event-triggering scheme for networked interconnected control system with stochastic uncertainty," International Journal of Robust and Nonlinear Control, vol. 27, no. 12, pp. 236-251, 2017.

[17] Z. Gu, E. Tian, and J. Liu, "Adaptive event-triggered control of a class of nonlinear networked systems," Journal of the Franklin Institute, vol. 354, no. 9, pp. 3854-3871, 2017.

[18] Q. Zhou, W. Wang, H. Ma et al., "Event-triggered fuzzy adaptive containment control for nonlinear multi-agent systems with unknown bouc-wen hysteresis input," IEEE Transactions on Fuzzy Systems, 2019.

[19] Q. Zhou, W. Wang, H. Liang et al., "Observer-based eventtriggered fuzzy adaptive bipartite containment control of multi-agent systems with input quantization," IEEE Transactions on Fuzzy Systems, 2019.

[20] H. Yan, H. Zhang, X. Zhan, Z. Li, and C. Yang, "Event-based \$h_\{linfty\}\$ fault detection for buck converter with multiplicative noises over network," IEEE Transactions on Circuits and Systems I: Regular Papers, vol. 66, no. 6, pp. 2361-2370, 2019.

[21] H. Shen, F. Li, H. Yan, H. R. Karimi, and H.-K. Lam, "Finitetime event-triggered $\$ \backslash$ mathcal $\{\mathrm{h}\} \_\{\text {infty }\} \$$ control for t-s fuzzy markov jump systems," IEEE Transactions on Fuzzy Systems, vol. 26, no. 5, pp. 3122-3135, 2018.

[22] A. Rahnama, M. Xia, and P. J. Antsaklis, "Passivity-based design for event-triggered networked control systems," IEEE Transactions on Automatic Control, vol. 63, no. 9, pp. 27552770, 2018.

[23] Z. Gu, P. Shi, D. Yue, and Z. Ding, "Decentralized adaptive event-triggered \$h_linfty\$ filtering for a class of networked nonlinear interconnected systems," IEEE Transactions on Cybernetics, vol. 49, no. 5, pp. 1570-1579, 2019.

[24] J. Qiu, K. Sun, T. Wang, and H. Gao, "Observer-based fuzzy adaptive event-triggered control for pure-feedback nonlinear systems with prescribed performance," IEEE Transactions on Fuzzy Systems, vol. 27, no. 11, pp. 2152-2162, 2019.

[25] K. Zhang, T. Zhao, and S. Dian, "Dynamic output feedback control for nonlinear networked control systems with a twoterminal event-triggered mechanism," Nonlinear Dynamics, vol. 100, no. 3, pp. 2537-2555, 2020.

[26] K. Gu, J. Chen, and V. Kharitonov, "Stability of time-delay systems,” Birkhauser Boston, vol. 5, no. 4, pp. 213-217, 2003.
[27] H.-B. Zeng, Y. He, M. Wu, and S.-P. Xiao, "Less conservative results on stability for linear systems with a time-varying delay," Optimal Control Applications and Methods, vol. 34, no. 6, pp. 670-679, 2013.

[28] H. B. Zeng, Y. He, and M. Wu, "Free-matrix-based integral inequality for stability analysis of systems with time-varying delay," IEEE Transactions on Automatic Control, vol. 60, no. 10, pp. 2768-2772, 2017.

[29] G. Chen, J. Xia, G. Zhuang, and J. Zhao, "Improved delaydependent stabilization for a class of networked control systems with nonlinear perturbations and two delay components," Applied Mathematics and Computation, vol. 316, pp. 1-17, 2018.

[30] N. Xu and L. Sun, "Parallel distributed compensation controller design for markovian jump system with time-varying delays using bessel-legendre inequality method and improved positive definite rule," Transactions of the Institute of Measurement and Control, vol. 42, no. 7, pp. 1313-1322, 2019.

[31] P. Chen and J. Zhang, "Event-triggered output-feedback ho control for networked control systems with time-varying sampling," Control Theory and Applications, vol. 9, no. 9, pp. 1384-1391, 2015.

[32] A. Seuret and F. Gouaisbaut, "Wirtinger-based integral inequality: application to time-delay systems," Automatica, vol. 49, no. 9, pp. 2860-2866, 2013.

[33] F. Li, J. Fu, and D. Du, "An improved event-triggered communication mechanism and L- $\infty$ control co-design for network control systems," Information Sciences, vol. 370, pp. 743-762, 2016.

[34] H. K. Khalil, Nonlinear Systems, Prentice-Hall, Upper Saddle River, NJ, USA, 3rd edition, 2002. 